\section{Suicide in ethnic minority groups ${ }^{\dagger}$}

\author{
KWAME MCKENZIE, MARC SERFATY and MICHAEL CR AWFORD
}

Suicide is a complex phenomenon with numerous influences, including the individual's personality, biology, culture and social environment, as well as the macroeconomic and political context.

In 2000 there were over 800000 suicides worldwide. The World Health Organization estimates that more people die each year from suicide than in all the world's armed conflicts. The rate of suicide has generally been increasing over the past 50 years and there has been a change in the age risk profile: those over 45 years old represented $60 \%$ of the total in 1950 but only $45 \%$ by 1998 . These rate changes are mainly due to fluctuations in the number of male suicides (World Health Organization, 2002).

\section{SUICIDE RATES IN DIFFERENT COUNTRIES}

The suicide rate varies internationally. Some South and Central American countries report annual rates as low as 3 per 100000 , whereas the former Soviet Union and eastern bloc countries have relatively high rates: Belarus and the Russian Federation both have male suicide rates of over 60 per 100000 (World Health Organization, 2002). Cultural factors influence the likelihood of unexplained deaths being classified as suicides, but such biases are not sufficient to explain national differences in rates or changing rates in countries over time. Similar economic and political changes have different effects on suicide rates in different countries, which underlines the importance of culture in modifying the effects of macroeconomic change (Makinen, 2000). The importance of culture is emphasised by international differences in the gender and age groups that are at highest risk, and in the relevance of particular risk factors (Ji et al, 2001). International

†See pp. 155-160, this issue. figures suggest that the UK and USA have mid-range suicide rates with annual death rates per 100000 of approximately 12 for men and 3 for women in the UK, and 18 and 4 respectively in the USA (World Health Organization, 2002).

\section{SUICIDE RATES IN ETHNIC GROUPS WITHIN COUNTRIES}

Aggregated figures hide major geographic discrepancies and variations in rates between ethnic groups within a country. For example, there are high rates in the aboriginal peoples of Australia and New Zealand compared with the colonists, and in a number of populations from the Indian diaspora around the world. The suicide rate in White Americans is double that of African Americans, but this is largely accounted for by the disproportionately higher rates in older White men. Native Americans (including those in Alaska) have rates of suicide that are at least $50 \%$ higher than the White population. East Asian American women have the highest suicide rates of all women over the age of 65 years in the USA. Hispanic Americans are half as likely to complete suicide as White Americans, but there are significantly higher rates of suicidal ideation and suicide attempts in young Hispanic Americans at high school which are now being reflected in their suicide rates. Changes in suicide rates have also been reported in African American youths aged 10-14 years: between 1980 and 1995 there was an increase almost double that for the White aged-matched population (US Department of Health and Human Services, 2001).

In the UK, place of birth rather than ethnicity is recorded on the death certificate, which makes national comparative studies from routine data-sets difficult. Half of the UK's ethnic minority population is born in the UK, and the problem is further compounded by the fact that these people tend to be younger and may be at increased risk of suicide compared with their parents. Soni Raleigh (1996) used data on place of birth to compare suicide ratios of foreignborn and UK-born nationals. Suicide ratios were significantly lower in men born in Bangladesh, Sri Lanka and Pakistan at all ages; they were raised in young men born in India and East Africa; and they were also significantly higher in Indian and East African women, in whom there was a two- to threefold excess at ages 15-34 years. Ratios were low in Pakistani and Bangladeshi women overall, but elevated in the 15- to 25-year-old group. The suicide ratios in those who were Caribbean-born were low overall, but raised in the 25- to 34-year-old group.

Neeleman \& Wessely (1999) conducted a survey of all unexplained deaths in one area of London. They found that residents of African-Caribbean origin had relatively low suicide rates and young Indian women relatively high rates. The rates of suicide in residents born in Scotland and Ireland were 2.1 to 2.9 times higher than the local base rate. In their study the suicide rates of young White men were higher than those of the elderly.

\section{RISK FACTORS FOR SUICIDE}

Individual risk factors for suicide are varied, but in many countries rates are highest in men, those who are divorced or separated, the unemployed, those in social class 5 and those who are socially isolated. The rates of suicide are highest in spring and early summer in both northern and southern hemispheres. Low risk is associated with religiosity. Alcohol consumption and easy access to toxic substances or firearms are positively correlated with suicide rates. The majority of those who kill themselves have a psychiatric illness, and this and recent admission to a psychiatric hospital are by far the most important risk factors at a population level.

Durkheim (1897) proposed that the societal and cultural context influenced the risk of suicide in an individual. $\mathrm{He}$ described three types of suicide: egoistic (individuals who had lost their sense of integration within their social group and so no longer felt subject to social, family or religious control); anomic (individuals who lived in a society that lacked collective order because it was in the midst of social change or political crisis); and altruistic 
(people who sacrificed their lives for the good of the community). Analyses of Durkheim's hypotheses using modern statistical methods have generally supported his theories. Rates of suicide are high during economic depression and low during war. In the USA, states that spend more on public welfare have lower suicide rates.

\section{RISK FACTORS FOR SUICIDE IN MINORITY GROUPS}

Recent Swedish studies have concluded that first-generation immigrants tend to have higher rates of suicide than the population of their country of origin, but the rate is higher still in their Swedish-born children (Hjern, 2002). In addition to cultural factors that mediate suicide rates in ethnic minority groups, the acceptability of suicide in the ethnic majority population and the response of the host culture to the ethnic minority group are important. Although it has been argued that ethnic minority groups will acquire the rates of suicide of the ethnic majority group over time, there have been persistently lower rates of suicide in some groups - for instance African Americans - and consistently higher rates in other ethnic minority populations, such as the native populations of the USA and Australia.

Neeleman et al (2001) demonstrated that suicide rates are higher in areas where ethnic minority groups are in lower concentration. This is not due to confounding by gender, age, deprivation or unbalanced migration. This effect may not be due to ethnicity per se, as other density effects have been demonstrated. For instance, there are higher rates of depression in single mothers who live in areas with a low concentration of single mothers than in those who live in areas with a higher proportion of single mothers (Driessen et al, 1998). It may be that a relative lack of support by people with similar social situations or the perception of a more hostile social environment explains these findings.

At an individual level, socio-economic stress, thwarted aspirations, racism, acculturation, culture clash with parents, loss of religious affiliation, difficulty with identity formation, and loss of family and community support may have effects on suicide risk. However, context is important; all of these risk factors are influenced by the socio-economic situation of the group, its culture and history, and the mainstream

KWAME MCKENZIE, BM, MRCPsych, MARC SERFATY, MPhil, MBChB, MRCPsych, Department of Psychiatry and Behavioural Sciences, Royal Free and University College Medical School, London; MICHAEL CRAWFORD, MSc, MB, MD, MRCPsych, Department of Psychological Medicine, Imperial College, London, UK

Correspondence: Dr Kwame Mc Kenzie, Department of Psychiatry and Behavioural Sciences, Royal Free and University College Medical School, Royal Free Campus, Rowland Hill Street, London NW3 2PF, UK

(First received 17 December 2002, final revision 18 March 2003, accepted 19 March 2003)

social environment. The institutional deconstruction, decreased collectivism, increased normlessness and helplessness and increased personal risk that characterise a post-modern society may increase the risk of depression in the whole population. This could interact with the particular situation of an ethnic minority group to lead to an increased risk of suicide.

\section{SUICIDE IN MENTAL HEALTH USERS: A TARGET FOR PREVENTION}

Current suicide prevention strategies in developed countries target high-risk populations (World Health Organization, 2002). Most people who die by suicide have previously been in contact with psychiatric services, which makes service users an obvious target group. Service users from minority ethnic groups could be considered an even higher risk group because of their increased rates of mental health problems, high rates of untreated mental health problems, and the poorer levels of compliance and satisfaction with services. Conflict with services and poor compliance are important risk factors for suicide in people with mental health problems (De Hert et al, 2001). Add to these the late presentation to services by members of some ethnic minority groups, the increasing rates of suicide in younger populations and the skewed age distribution of ethnic minorities towards younger age groups, and it is clear that suicide in young ethnic minority service users is likely to be an increasingly important public health issue.

There has been no specific investigation of risk factors for suicide in ethnic minority mental health service users in the UK. It remains unclear who is at increased risk, and why. The paper by Hunt et al (2003, this issue) goes some way to filling this void, but information from their report is limited and more in-depth work will be needed if we are to be able to predict who is at risk. For instance, Hunt et al report that clinicians believe that suicides in young
African-Caribbean service users are more likely to be preventable, but we do not yet have the data to target those at greatest risk or to develop evidence-based prevention strategies.

\section{DECLARATION OF INTEREST}

None.

\section{REFERENCES}

De Hert, M., McKenzie, K. \& Peuskens, J. (200I) Risk factors for suicide in young people suffering from schizophrenia: a long term follow-up study. Schizophrenia Research, 47, 127-134.

Driessen, G., Gunther, N. \& Van Os, J. (1998) Shared social environment and psychiatric disorder: a multilevel analysis of individual and ecological effects. Social Psychiatry and Psychiatric Epidemiology, 33, 606-612.

Durkheim, E. (1897) Le Suicide. Trans. J. H. Spalding \& G. Simpton (195I). New York: Free Press.

Hjern, A. (2002) Suicide in first and second generation immigrants in Sweden: a comparative study. Social Psychiatry and Psychiatric Epidemiology, 37, 423-429.

Hunt, I. M., Robinson, J., Bickley, H., et al (2003) Suicides in ethnic minorities within 12 months of contact with mental health services. National clinical survey. British Journal of Psychiatry, 183, 155-160

Ji, J., Kleinman, A. \& Becker, A. E. (200I) Suicide in contemporary China: a review of China's distinctive suicide demographics in their socio-cultural context. Harvard Review of Psychiatry, 9, 1-12.

Makinen, I. H. (2000) Eastern European transition and suicide mortality. Social Science and Medicine, 5I, 1405-1420.

Neeleman, J. \& Wessely, S. (1999) Ethnic minority suicide: a small area geographical study in south London. Psychological Medicine, 29, 429-436.

_ ,Wilson-Jones, C. \& Wessely, S. (200I) Ethnic density and deliberate self harm; a small area study in south east London. Journal of Epidemiology and Community Health, 55, 85-90.

Soni Raleigh, V. (1996) Suicide patterns and trends in people of Indian subcontinent and Caribbean origin in England and Wales. Ethnicity and Disease, I, 55-64.

US Department of Health and Human Services (200I) Mental Health: Culture, Race and Ethnicity - A Supplementto Mental Health: A Report of the Surgeon General. Rockville, MD: USDepartment of Health and Human Services, Substance Abuse and Mental Health Services Administration, Centre for Mental Health Services.

World Health Organization (2002) Accessed I2/I2/ 2002. Mental health; Suicide prevention; SUPRE; Global charts; Country report. http: //www5.who.int/ mental_health 